\title{
Sistema Experto para la Rotación de Cultivos en un Invernadero
}

\section{Expert System for the rotation of crops in a greenhouse}

\author{
RAFAEL-PEREZ, Eva †*, MORALES-HERNANDEZ, Maricela, REYES-MORALES, Idalia Sarai y \\ NAVARRETE-INFANTE, Néstor Manuel
}

Instituto Tecnológico de Oaxaca, Departamento de Sistemas y Computación. Avenida Ing. Víctor Bravo Ahuja No. 125 Esquina Calzada Tecnológico, C.P. 68030

ID 1 ${ }^{\mathrm{er}}$ Autor: Eva, Rafael-Perez / ORC ID: 0000-0003-2793-1254, CVU CONACYT ID: 905268

ID $1^{\mathrm{er}}$ Coautor: Maricela, Morales-Hernandez, ORC ID: 0000-0002-3521-2041, CVU CONACYT ID: 731036

ID $2^{\text {do }}$ Coautor: Idalia Sarai, Reyes-Morales / ORC ID: 0000-0003-3485-6985, CVU CONACYT ID: 999080

ID $3^{\text {er }}$ Coautor: Néstor Manuel, Navarrete-Infante / ORC ID: 0000-0002-0170-5622, CVU CONACYT ID: 337188

DOI: $10.35429 /$ JOIE.2019.9.3.1.11

Recibido Enero 27, 2019; Aceptado Marzo 30, 2019

\section{Resumen}

La producción de cultivos bajo invernadero es una de las técnicas más modernas que se utilizan actualmente en la producción agrícola. La rotación de cultivos es una práctica agrícola para aumentar la productividad de la tierra y optimizar el uso de recursos alternando diferentes tipos de cultivos en el mismo suelo. El proyecto denominado "Sistema Experto para la Rotación de Cultivos en un Invernadero" fue desarrollado con el objetivo de tomar la decisión del tipo de cultivo idóneo a sembrar en el invernadero "San Sebastián", ya que el riesgo de pérdida de producción se reduce considerablemente, de manera que ayude al productor a saber cuál es el cultivo idóneo a sembrar según la experiencia de un experto en la rama; mediante el uso de sensores se obtiene el registro de las temperaturas de ambiente y humedad en tiempo real; el software fue desarrollado utilizando el modelo de Prototipos.

Sistema experto, Rotación de cultivos, Invernadero

\begin{abstract}
The production of greenhouse crops is one of the most modern techniques currently used in agricultural production. Crop rotation is an agricultural practice to increase the productivity of the land and optimize the use of resources by alternating different types of crops in the same soil. The project called "Expert System for the rotation of crops in a greenhouse" was developed with the aim of making the decision of the type of suitable crop to be planted in the "San Sebastian" greenhouse. Since the risk of production loss is considerably reduced, so that it helps the producer to know which is the suitable crop to sow according to the experience of an agricultural expert; using physical sensors, the registration of ambient and humidity temperatures is obtained in real time; the software was developed by the prototype model.
\end{abstract}

Expert system, Rotation of crops, Greenhouse

Citación: RAFAEL-PEREZ, Eva, MORALES-HERNANDEZ, Maricela, REYES-MORALES, Idalia Sarai y NAVARRETE-INFANTE, Néstor Manuel. Sistema Experto para la Rotación de Cultivos en un Invernadero. Revista de Ingeniería Innovativa. 2019. 3-9: 1-11

\footnotetext{
*Correspondencia al Autor (Correo Electrónico: evarafaelperez@gmail.com)

$\dagger$ Investigador contribuyendo como primer autor.
} 


\section{Introducción}

La incorporación de las Tecnologías de la Información y la Comunicación (TIC) han transformado las condiciones y la manera de vida de la población rural, actividad que sigue siendo decisiva para la evolución de la humanidad, la forma de producción en la agricultura ha cambiado; los sistemas productivos han incrementado la productividad y la competitividad, mediante la automatización en los procesos de producción en los invernaderos de manera inteligente.

El proyecto Sistema experto para la rotación de cultivos en un invernadero fue desarrollado con el objetivo de apoyar al productor en la toma de decisión del tipo de cultivo idóneo a sembrar en el invernadero "San Sebastián", ubicado en la comunidad de San Sebastián Atoyaquillo en la región Mixteca del estado de Oaxaca, México.

Actualmente la agricultura considera diversos métodos de producción, eficientes y de alto rendimiento, una de ella es la agricultura protegida, que se define como un sistema agrícola especializado en el cual se lleva a cabo un cierto control del medio climático, alterando sus condiciones: suelo, temperatura, radiación solar, viento, humedad y composición atmosférica (Castellanos-Ramos, 2004).

La producción de cultivos bajo invernadero es una de las técnicas más modernas que se utilizan actualmente en la producción agrícola. Un invernadero provee un ambiente apropiado, tanto en el suelo como en el aire para el cultivo de especies vegetales.

La rotación de cultivos es una práctica agrícola para aumentar la productividad de la tierra y optimizar el uso de recursos alternando diferentes tipos de cultivos en el mismo suelo, su utilización contribuye a reducir la "fatiga" de los suelos; es decir, la disminución del rendimiento del cultivo debido a factores fitopatológicos, nutricionales, de malos manejos o alelopáticos; por lo que no sólo resuelve problemas de insectos y plagas, sino también es una solución económicamente viable. (Seminis, 2016).
El principio básico de las rotaciones es que los cultivos se suceden en función de las características entre el cultivo precedente y el siguiente, esperando algunos años a volver a plantar un determinado cultivo en la misma parcela, a la hora de diseñar una rotación, se deben combinar cultivos de características diferentes, siempre teniendo en cuenta el efecto que cada cultivo produce sobre la fertilización y la estructura del suelo.

Para la rotación de cultivos existen varias técnicas y se pueden simplificar o hacer más complejos según nuestras necesidades y experiencia, para este proyecto se basó en la técnica de rotación de 4 grupos 8 familias, iniciando con la familia de las Solanáceas, después la familia de las Leguminosas, posteriormente la familia Compuestas y finalmente la familia de las Umbelíferas.

Otro aspecto fundamental de la rotación de cultivos es la determinación de las reglas de rotación, ya que es importante tener en cuenta la familia de las plantas para saber qué tipo de suelo es requerido por cada planta, como para sus asociaciones con otras plantas beneficiosas para su crecimiento, ya que las necesidades nutricionales de las plantas varían principalmente según su familia.

Hoy en día la Inteligencia Artificial es la rama de la computación con mayor crecimiento en los últimos años, una de sus áreas son los Sistemas Expertos (SE), según (Velázquez \& Coca 2004) plantean que son un tipo de programa de aplicación informática que adopta decisiones o resuelve problemas de un determinado campo, como la agricultura, finanzas medicina entre otros, utilizando los conocimientos y reglas analíticas y definidas por los expertos en ese campo; se apoya de la base de conocimiento que proporciona hechos, objetivo y reglas del tema y del motor de inferencia que proporciona la capacidad de razonamiento que permite al sistema experto extraer conclusiones. El uso de los sensores, en la actualidad ha revolucionado la manera en que los agricultores hacen uso de ellos, debido a que un invernadero es de suma importancia conocer el estado de las variables climatológicas que se presenten como la temperatura y humedad para tener los datos precisos de las variables que monitorean que favorece a los productores, tanto económica como productivamente. 
En el estado de Oaxaca existe un crecimiento ascendente en la producción de cultivos bajo invernaderos, en la región mixteca la agricultura es la actividad básica generalmente de autoconsumo, los principales productos que se obtienen son maíz, frijol, trigo, ajo, tomate y cebolla; y que en muchas zonas de la región ya cuentan con la agricultura protegida en la producción de cultivos.

El invernadero San Sebastián es una empresa $100 \%$ mexicana, que está orientada a la producción y comercialización de cultivos bajo invernadero, para otorgarles a las familias oaxaqueñas un producto sano y de calidad.

El Sistema experto para la rotación de cultivos en un invernadero comprende varios módulos como son: cultivo, siembra cosecha, rotación, riego, bitácora, ver bitácora e historial, temperatura y humedad.

El presente trabajo se compone de los siguientes apartados: Metodología, planteamiento del problema, tipos de usuarios, desarrollo, resultados, conclusiones y referencias.

\section{Metodología}

Para la creación de software existen diferentes metodologías de desarrollo de software a seguir; para el proyecto denominado sistema experto para la rotación de cultivos en un invernadero, se utilizó la metodología basada en prototipos que tiene como objetivo principal la participación directa del cliente en la construcción del software requerido, ayuda a los participantes a mejorar la comprensión de lo que hay que elaborar cuando los requerimientos no están claros, la clave del modelo está en definir desde el principio las reglas del juego; es decir, todos los participantes deben estar de acuerdo en que el prototipo sirva como el mecanismo para identificar y definir los requerimientos del software, (Pressman, 2010, p. 37).

Sus fases son: comunicación, plan rápido, modelado diseño rápido, construcción del prototipo, despliegue, entrega y retroalimentación, ver figura 1, para este proyecto se realizaron tres prototipos, que fueron desarrollados y evaluados por parte del usuario final.

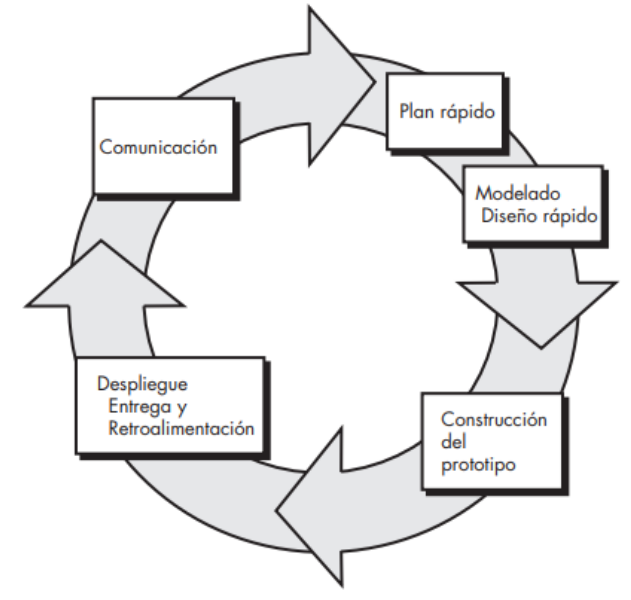

Figura 1 Modelo en prototipos

Fuente. (Pressman, 2010, p. 37)

\section{Planteamiento del problema}

La producción de cultivos bajo invernadero es una de las técnicas más modernas que se utilizan actualmente en la producción agrícola, uno de los principales problemas de cualquier invernadero es que son de monocultivo, es decir, solo siembran un tipo de cultivo, en el caso del invernadero "San Sebastián" casi siempre se ha sembrado jitomate, lo cual resulta a veces perjudicial para tanto para el mismo invernadero como para el productor; en los sistemas de monocultivo, con el paso del tiempo se observa un incremento de insectos, plagas $y$ enfermedades específicas del cultivo, asimismo, la cantidad de nutrientes disminuye, porque las plantas ocupan siempre la misma zona de raíces y en la temporada siguiente las raíces no se desarrollan bien según (SAGARPA, 2018), existe la "fatiga" de los suelos; es decir, la disminución del rendimiento del cultivo debido a factores fitopatológicos, y como consecuencia se presenta la pérdida del cultivo lo que genera pérdidas económicas para el productor.

\section{Tipos de usuario}

Para este sistema se identificaron 3 tipos de usuarios con diferentes roles y privilegios para el acceso al sistema. El usuario Administrador del sistema, tiene el acceso total a cada uno de los módulos, es el encargado de agregar datos al sistema de usuarios, nuevos cultivos y nuevos riegos. 
El usuario administrador del invernadero agrega los datos relacionado al invernadero de siembras, cosechas, bitácora de procesos y solo visualiza la información de los riegos, cultivos, historial de siembras y cosechas, rotación de cultivos, bitácora de procesos; y el usuario invitado solo puede ver información de los riegos, cultivos, historial de siembras y cosechas, rotación de cultivos, bitácora de procesos.

\section{Desarrollo}

Para el desarrollo del proyecto, se realizaron tres tres prototipos que le permitieron la adecuación de los requisitos del usuario en el sistema, por lo tanto, en cada uno de prototipos se realizaron las fases de comunicación, plan rápido, modelado diseño rápido, construcción del prototipo, despliegue, entrega y retroalimentación del sistema que a continuación se describen:

En la fase de Comunicación, se realizaron las diferentes entrevistas con el productor del invernadero para conocer cuáles son las necesidades reales y los problemas presentados para generar los requerimientos del sistema, por ejemplo: actividades y procesos manuales, cantidad de camas, altura, longitud y la distancia entre una cama y otra, sistema de riego, preparación manual de abono, fertilización, periodos de siembra, de cosecha, corte, condiciones climatológicas en cuanto a humedad y temperatura, tipo de cultivos que conoce, cantidad de cosecha que obtuvo de ellas para definir los objetivos generales del software, identificar los requerimientos y detectar las áreas en las que es imprescindible una mayor definición.

Para la definición de requerimientos se utilizó la técnica de casos de uso, para especificar el comportamiento del sistema. Un caso de uso narra una historia estilizada sobre cómo interactúa un usuario final (que tiene cierto número de roles posibles) con el sistema en circunstancias específicas, ilustra el software o sistema desde el punto de vista del usuario final (Pressman, 2010, p.113). A continuación, se muestra un ejemplo de caso de uso del usuario administrador del sistema, ver figura 2.

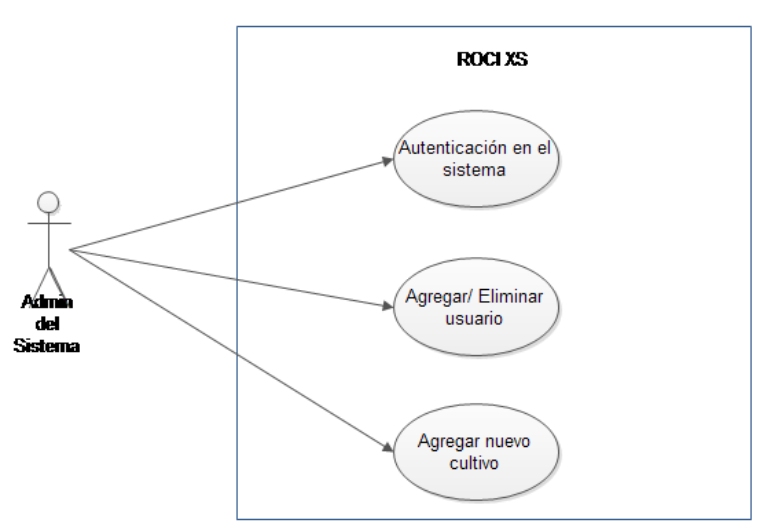

Figura 2 Caso de uso de administrador del sistema Fuente: Elaboración Propia

En la fase del Plan rápido se generó un plan de prototipos donde especifica los módulos, las actividades a realizar en cada uno de ellos, y la manera en que interactúan los usuarios con el sistema a través de las interfaces ver tabla 1.

\begin{tabular}{|c|c|}
\hline & Primer Prototipo: \\
\hline & $\begin{array}{l}\text { Autenticación en el sistema } \\
\text { Usuarios } \\
\text { Módulo de siembra } \\
\text { Módulo de cosecha } \\
\text { Ver historial de siembras y cosechas } \\
\text { Ver cultivos } \\
\text { Dar de alta, visualizar, mostrar, editar }\end{array}$ \\
\hline & Segundo Prototipo: \\
\hline & $\begin{array}{l}\text { Agregar riego para cultivos } \\
\text { Insertar descripción del proceso (Bitácora) } \\
\text { Ver bitácora } \\
\text { Ver Riego } \\
\text { Módulo de rotación } \\
\text { Ingresar mes } \\
\text { Ver cultivo siguiente } \\
\text { Seleccionar cultivo siguiente } \\
\text { Analiza la secuencia de rotación } \\
\text { Reglas de rotación } \\
\text { Analiza temperaturas } \\
\text { Interacción de usuarios con las interfaces: Ingresa } \\
\text { cultivo actual, Ingresa cultivo anterior1, Ingresa } \\
\text { cultivo anterior2 }\end{array}$ \\
\hline & Tercer prototipo: \\
\hline & $\begin{array}{l}\text { Módulo de humedad } \\
\text { Módulo de temperatura } \\
\text { Arduino/sensores } \\
\text { Lectura de temperatura y humedad } \\
\text { Temperaturas mínimas y máximas } \\
\text { Obtener humedades mínimas y máximas } \\
\text { Agregar o actualizar temperaturas y humedades en la } \\
\text { base de datos } \\
\text { Ver temperatura actual } \\
\text { Ver humedad actual }\end{array}$ \\
\hline
\end{tabular}

Tabla 1 Plan de prototipos Fuente: Elaboración Propia 
La fase de Modelado diseño rápido, es fundamental ya que se crean los diferentes modelos que sirve de base para el correcto funcionamiento del sistema, se diseñaron los diagramas de secuencia, la base de datos y las interfaces correspondientes a cada uno de los prototipos, a manera de ejemplo se muestra un diagrama de secuencia que modela la secuencia y la interacción del usuario con el sistema, la interfaz y la base de datos, ver figura 3 .

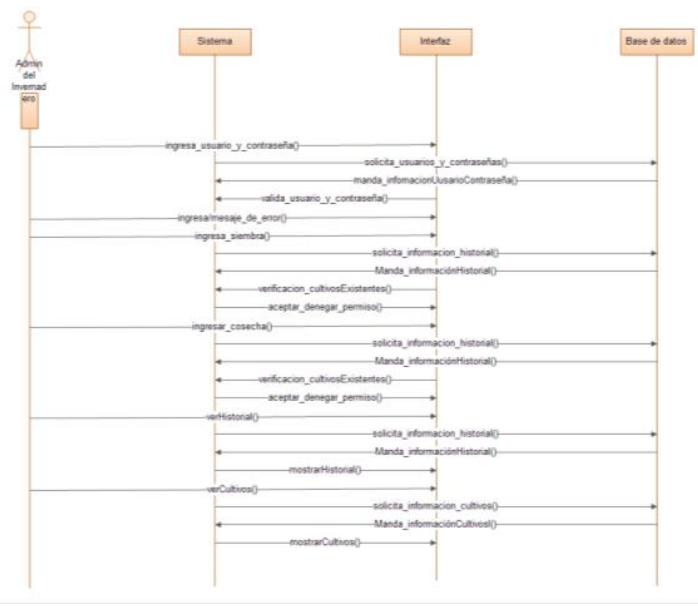

Figura 3 Diagrama de secuencia Administrador Fuente: Elaboración Propia

La figura 4, muestra el modelo de base de datos, es importante mencionar que para la creación de la base de datos se utilizó el sistema gestor de base de datos MySQL

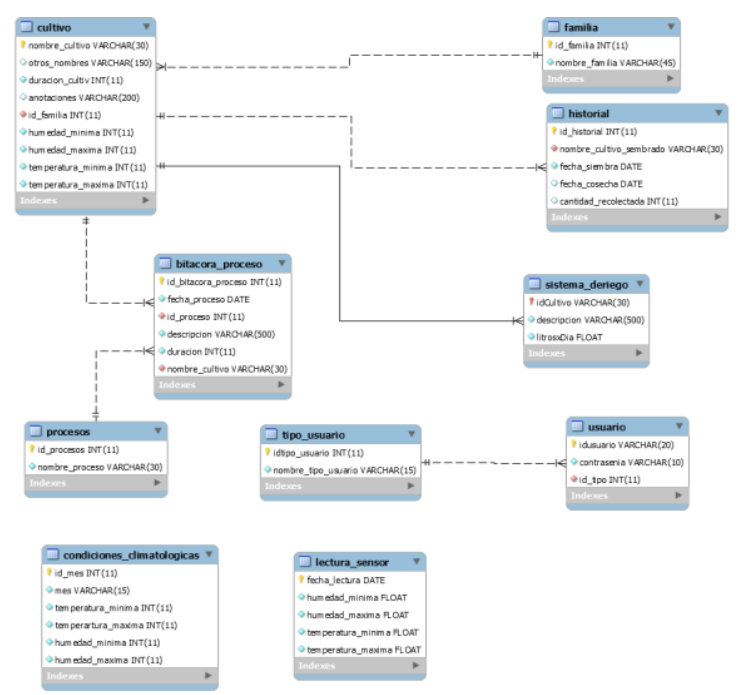

Figura 4 Modelo de base de datos relacional Fuente: Elaboración Propia

A continuación se muestran las interfaces de pantallas con diseño simples, ver figura 5 y 6 .

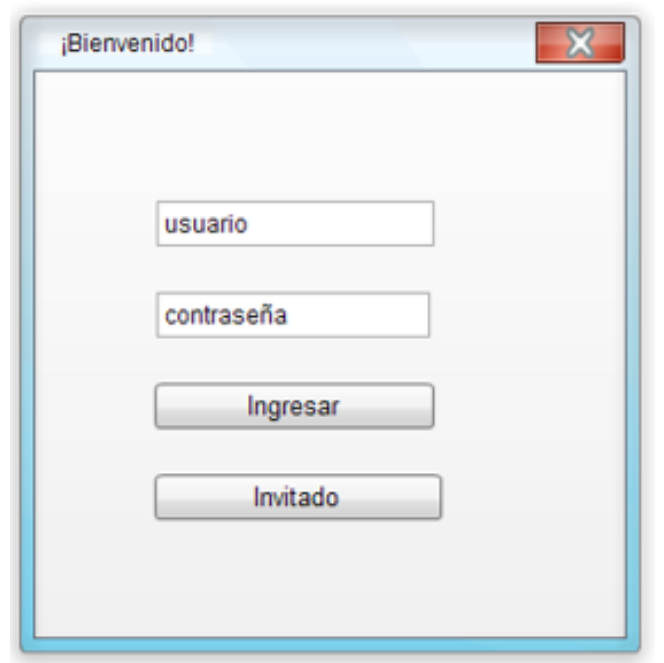

Figura 5 Acceso al sistema Fuente: Elaboración Propia

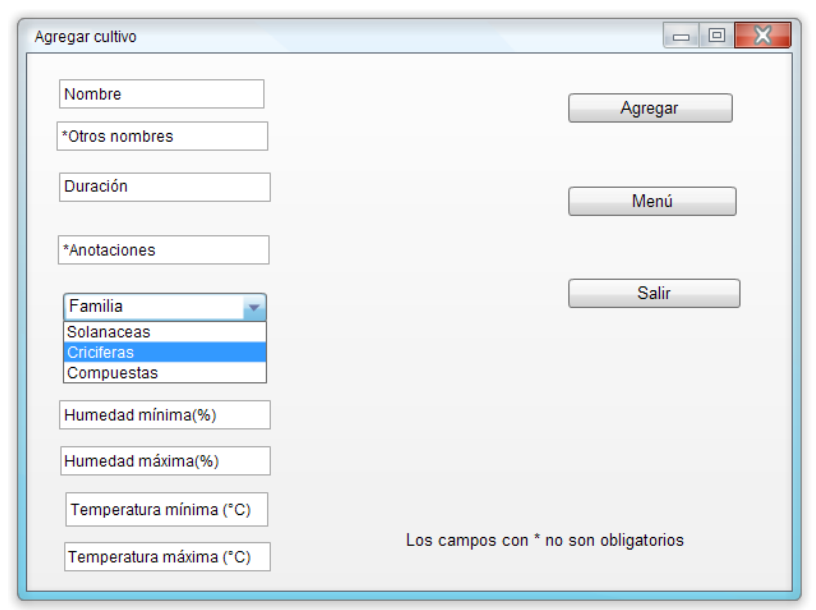

Figura 6 Agregar cultivos Fuente: Elaboración Propia

El diseño rápido lleva a la construcción de un prototipo. Éste se entrega y es evaluado por los participantes, que dan retroalimentación para mejorar los requerimientos; para este proyecto se utilizó el lenguaje de programación en Java, utilizando la librería de Eclipse IDE for Java Developers, que es una plataforma de desarrollo de código abierto basada en Java; y que se realizó en forma paralela con el diseño, es importante decir que los resultados de la construcción de cada uno de los prototipos está en función de las iteraciones.

La iteración ocurre a medida de que el prototipo es afinado para satisfacer las necesidades de distintos participantes, y al mismo tiempo le permite al usuario entender mejor lo que se necesita hacer. 
En la última fase que es Despliegue, entrega y retroalimentación del sistema, se aplicaron diferentes tipos de pruebas, por ejemplo las pruebas de integración, donde se comprobó que los módulos y elementos que interactúan en el sistema funcionen de manera correcta; pruebas de contenido donde se verificó que los botones usados para enlazar a más contenidos fueran los correctos e intuitivos para el usuario; y finalmente los prototipos fueron evaluados por los diferentes usuarios y se realizaron las modificaciones correspondientes así como la retroalimentación necesaria del sistema solicitado por el productor.

\section{Rotación de cultivos}

El Sistema Experto para la Rotación de Cultivos en un Invernadero fue desarrollado con el objetivo de tomar la decisión del tipo de cultivo idóneo a sembrar en el invernadero "San Sebastián", ya que el riesgo de pérdida de producción se reduce considerablemente, de manera que ayude al productor a saber cuál es el cultivo idóneo a sembrar según la experiencia de un experto.

Este proyecto se basó en la técnica de rotación de 4 grupos 8 familias y su ciclo de rotación inicia con el primer cultivo de las solanáceas, una familia exigente ya que requiere gran cantidad de nutrientes, especialmente nitrógeno(N), para ello, si no se dispone de buen suelo rico en materia orgánica, conviene añadírsela, abonando antes de la siembra; una vez hemos finalizado con las solanáceas, se sugiere plantar leguminosas o crucíferas. Las dos familias son consideradas como mejoradoras. Esto se debe principalmente a sus características en relación al suelo.

Las leguminosas, mantienen una simbiosis en el suelo con microorganismos del género Rhizobium, que son fijadores del Nitrógeno atmosférico y lo incorporan al suelo. Por su parte, las crucíferas, son plantas que mejoran la estructura del suelo por su raíz pivotante $\mathrm{y}$, además, reincorporan el azufre $(\mathrm{S})$ al suelo. El siguiente a sembrar son los de los cultivos "mejoradores", se plantan cultivos de exigencia media, como pueden ser las compuestas, las quenopodiáceas o las cucurbitáceas.
Finalmente, encontramos las umbelíferas y las liliáceas, ambos cultivos poco exigentes a nivel nutricional y que dejan el suelo preparado para abonar nuevamente y volver a empezar el ciclo de rotación. (Pellicer, 2015), ver figura 7.

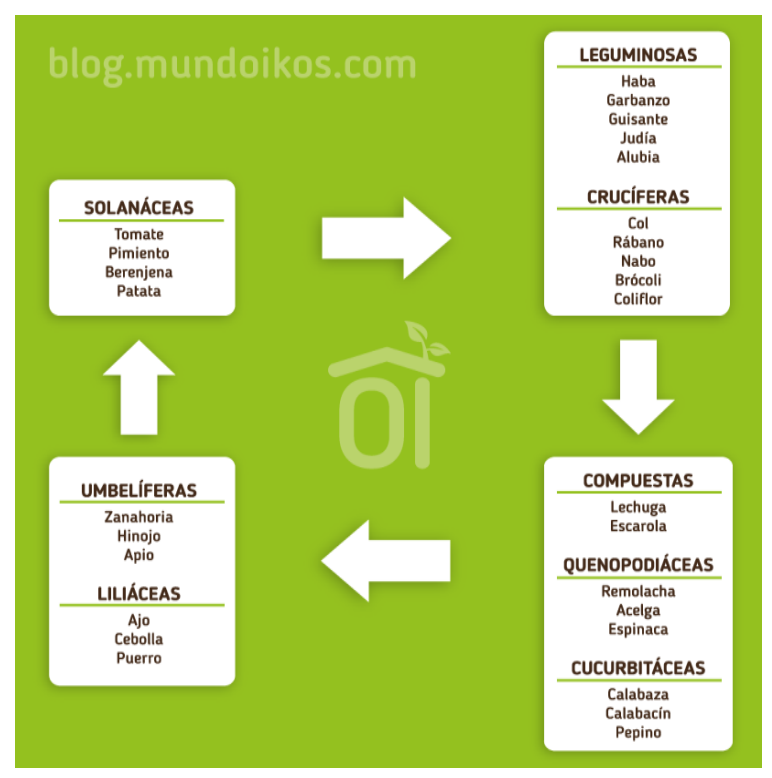

Figura 7 Rotación de cultivos, 4 grupos 8 familias Fuente: (Pellicer, 2015) Recuperado de https://blog.mundoikos.com/familias-de-cultivos-yrotaciones/

Para la rotación de cultivos de este sistema experto se han tomado 8 familias de cultivos que serán dividas en cuatro grupos, tal como se ve en la tabla 2, llamaremos al grupo sombreado con el color verde como el grupo "A", el sombreado por el color azul como el grupo "B", el amarillo como grupo "C" y el gris como el grupo " $\mathrm{D}$ ".

\begin{tabular}{|l|l|}
\hline Solanácea & Leguminosa \\
\cline { 2 - 2 } & Crucífera \\
\hline \multirow{2}{*}{ Umbelífera } & Compuestas \\
\cline { 2 - 2 } & Quenopodiácea \\
\hline Liliácea & Cucurbitácea \\
\hline
\end{tabular}

Tabla 2 Clasificación de familias

La rotación se hará de la siguiente manera:

Familia $\mathrm{A} \rightarrow$ Familia $\mathrm{B} \rightarrow$ Familia $\mathrm{C} \rightarrow$ Familia $\mathrm{D} \rightarrow$ Familia A...

\begin{tabular}{|l|l|l|}
\hline Actual & \multicolumn{1}{l|}{ Anterior } & Siguiente \\
\hline Solanácea & Leguminosa & Crucífera \\
\hline Solanácea & Crucífera & Leguminosa \\
\hline Solanácea & & Crucífera \\
\hline
\end{tabular}

Tabla 3 Cultivo siguiente del grupo A 
Determinar el cultivo siguiente después de un cultivo del grupo A, va a requerir del nombre de la familia del cultivo actual, así como el nombre de la familia del cultivo ya sea de la familia de las umbelíferas o liliáceas anterior, quedando de la siguiente manera.

\begin{tabular}{|l|l|l|l|}
\hline \multicolumn{2}{|c}{ Actual Anterior } & \multicolumn{1}{c|}{ Anterior2 } & \multicolumn{1}{c|}{ Siguiente } \\
\hline Leguminosa & Compuesta & Quenopodiácea & Cucurbitácea \\
\hline Leguminosa & Quenopodiácea & Cucurbitácea & Compuesta \\
\hline Leguminosa & Cucurbitácea & Compuesta & Quenopodiácea \\
\hline Crucífera & Compuesta & Quenopodiácea & Cucurbitácea \\
\hline Crucífera & Quenopodiácea & Cucurbitácea & Compuesta \\
\hline Crucífera & Cucurbitácea & Compuesta & Quenopodiácea \\
\hline Leguminosa & & & compuesta \\
\hline Leguminosa & Compuesta & & Cucurbitácea \\
\hline Leguminosa & Quenopodiácea & & Cucurbitácea \\
\hline Leguminosa & Cucurbitácea & & Compuesta \\
\hline Crucífera & & & compuesta \\
\hline Crucífera & Compuesta & & Cucurbitácea \\
\hline Crucífera & Quenopodiácea & & Cucurbitácea \\
\hline Crucífera & Cucurbitácea & & Compuesta \\
\hline
\end{tabular}

Tabla 4 Cultivo siguiente del grupo B

Para determinar el cultivo siguiente después de un cultivo que pertenece al grupo $\mathrm{B}$, se requiere del cultivo actual, y los dos anteriores cultivos pertenecientes al grupo $\mathrm{C}$, esto para evitar sembrar la misma familia en un lapso de tiempo mayor.

\begin{tabular}{|l|l|l|}
\multicolumn{1}{c}{ Actual } & \multicolumn{1}{c|}{ Anterior } & \multicolumn{1}{c|}{ Siguiente } \\
\hline Compuesta & Umbelífera & Liliácea \\
\hline Compuesta & Liliácea & Umbelífera \\
\hline Quenopodiácea & Umbelífera & Liliácea \\
\hline Quenopodiácea & Liliácea & Umbelífera \\
\hline Cucurbitácea & Umbelífera & Liliácea \\
\hline Cucurbitácea & Liliácea & Umbelífera \\
\hline Compuesta & & Umbelífera \\
\hline Quenopodiácea & & Umbelífera \\
\hline Cucurbitácea & & Umbelífera \\
\hline
\end{tabular}

Tabla 5 Cultivo siguiente del grupo C

Para determinar el cultivo siguiente después de un cultivo que pertenece al grupo $\mathrm{C}$, se requiere del cultivo actual, y el anterior cultivo pertenecientes al grupo $\mathrm{D}$, esto para evitar sembrar la misma familia en un lapso de tiempo mayor.

\begin{tabular}{|l|c|}
\hline \multicolumn{1}{|c|}{ Anterior } & Siguiente \\
\hline Liliácea & Solanácea \\
\hline Umbelífera & Solanácea \\
\hline
\end{tabular}

Tabla 6 Cultivo siguiente del grupo D

En el caso de los cultivos del grupo D, solo se requiere del cultivo actual para determinarnos el cultivo siguiente.
Por último, una última regla es que, si no se encuentra un cultivo actual y por consiguiente tampoco se encuentra un cultivo anterior, la regla nos dirá que el cultivo que se seleccionara será alguno de la familia de las solanáceas

\section{Resultados}

Para este sistema se implementaron diferentes módulos que fueron retroalimentados $\mathrm{y}$ evaluados para satisfacer las necesidades de los usuarios. A continuación se describen los módulos:

Acceso al sistema; se otorgaron los permisos de acceso a la información de acuerdo a los tipos de usuario, para ingresar al sistema cada usuario cuenta con un nombre de usuario y una contraseña. Ver Figura 8.

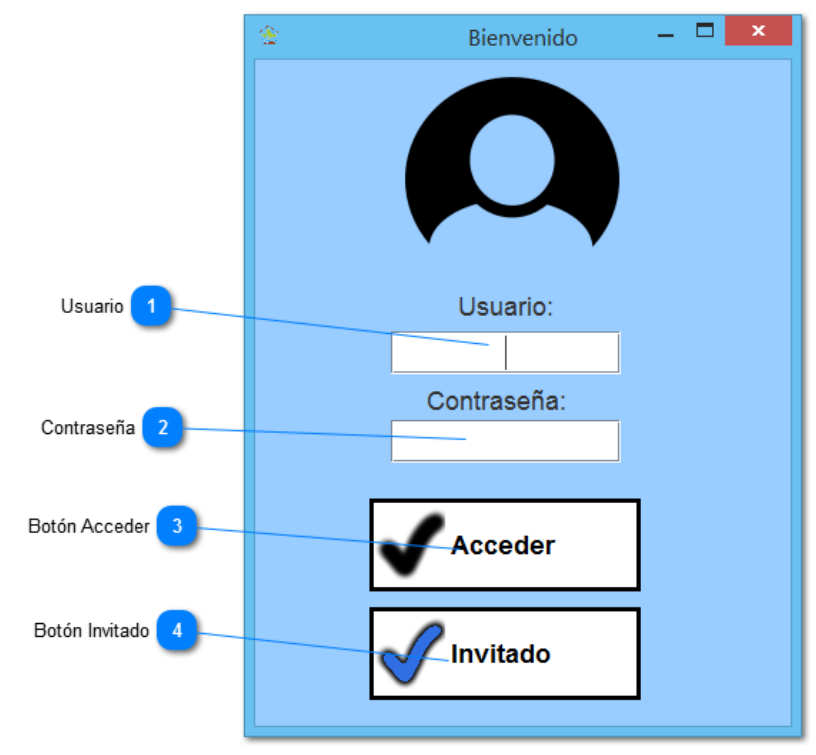

Figura 8 Pantalla de Acceso al sistema experto Fuente: Elaboración Propia

\section{Navegación en el Sistema.}

Al iniciar la sesión de forma correcta, muestra la pantalla principal del sistema que se compone del título, menú de opciones, e interfaz del usuario, como ejemplo se observa la pantalla principal del usuario administración del invernadero en la figura 9. 


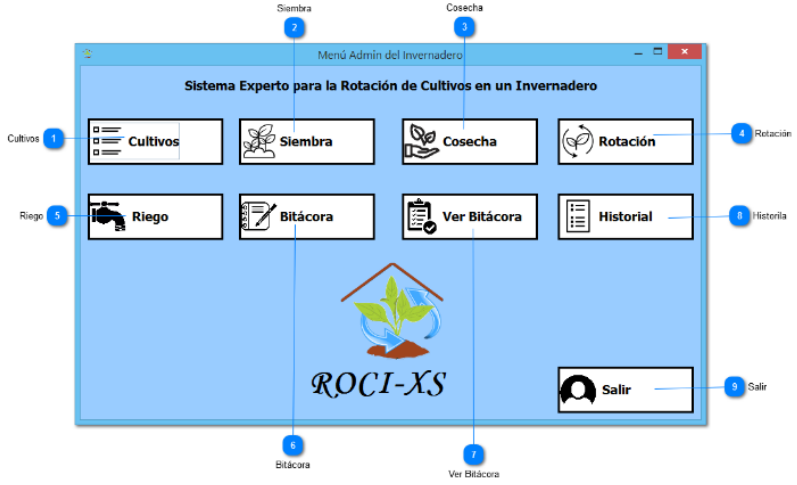

Figura 9 Pantalla Principal del sistema Fuente: Elaboración Propia

En el módulo de cultivos, se agregan nuevos cultivos a la base de datos, de existir en el sistema mandará un mensaje con la leyenda de "Este cultivo ya existe en la base de datos", es necesario rellenar los datos obligatorios, de lo contrario no se podrá ejecutar la acción, en el caso de las temperaturas y humedades solo se puede agregar números, ver figura 10.

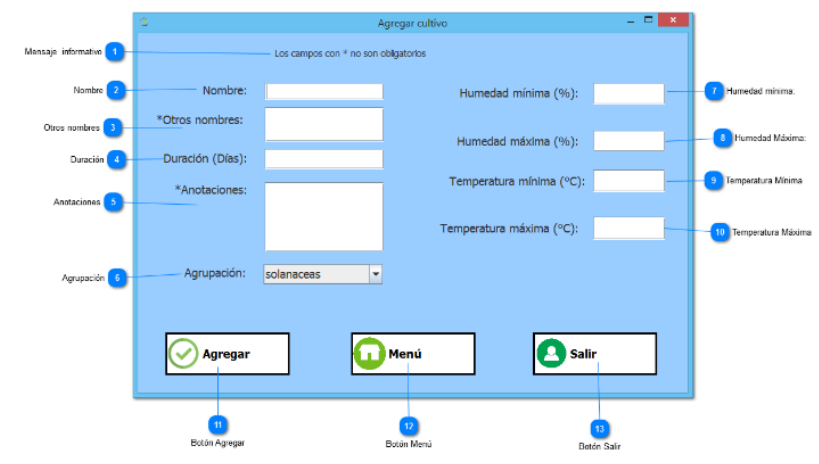

Figura 10 Agregar nuevo cultivo

Fuente: Elaboración Propia

La figura 11, contiene todos los cultivos existentes en la base de datos y al dar clic sobre alguno de ellos se muestra en los campos de la parte inferior la información del cultivo seleccionado, en caso de que los campos que no son obligatorios no contengan información se despliega la leyenda "Sin registro".

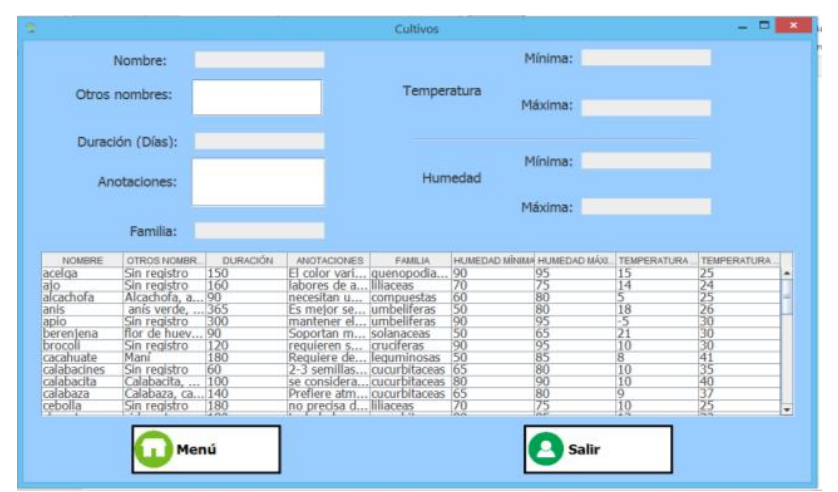

Figura 11 Agregar nuevo cultivo

Fuente: Elaboración Propia
Módulo de registrar siembra; para poder registrar una nueva siembra, es importante, tener la fecha de la última cosecha ya registrada para que el nombre de la siembra sea aceptado como valido este debe pertenecer al campo nombre que se muestra en la ventana de cultivos. Ver figura 12.

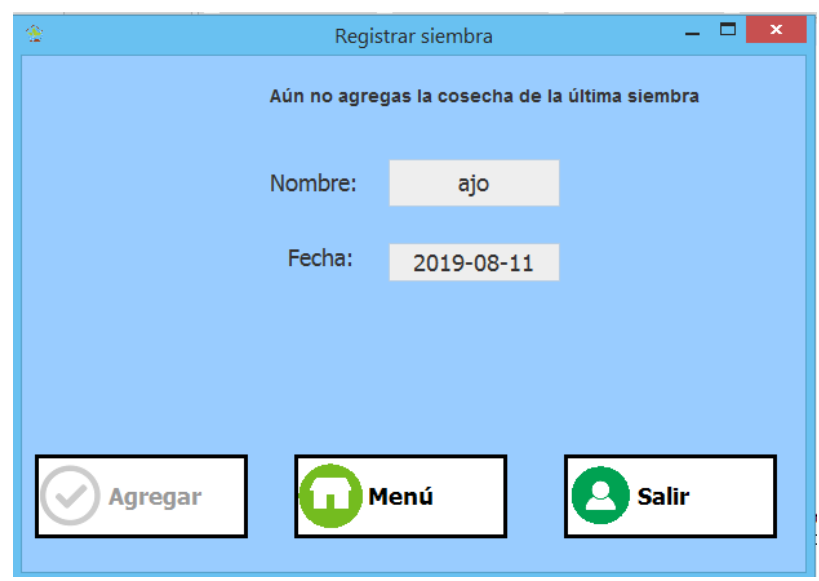

Figura 12 Registrar nueva siembra Fuente: Elaboración Propia

Módulo de cosecha. Para registrar una nueva cosecha y se cuente con una siembra que aún no registra la cosecha, los campos nombre y cosecha serán rellenados automáticamente con la información de la última siembra dejando los campos cosecha y cantidad recolectadas editables para que el usuario pueda ingresar la información correspondiente, la fecha de cosecha debe ser posterior a la fecha de la siembra de lo contrario no se podrá guardar la cosecha, como se muestra en la figura 13.

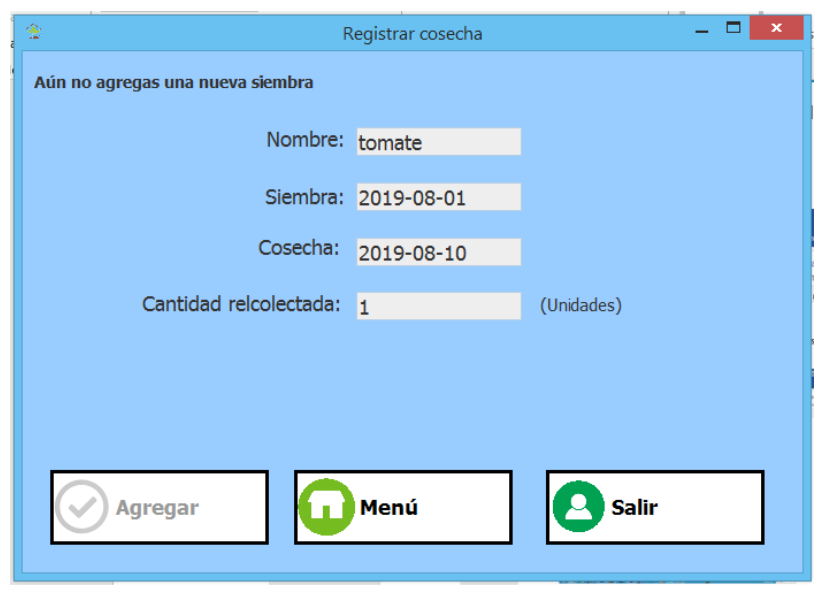

Figura 13 Agregar nueva cosecha Fuente: Elaboración Propia

Módulo de rotación de cultivos, este módulo es fundamental para el adecuado funcionamiento del sistema experto, existen campos que son rellenados automáticamente. 
Este campo se rellena automáticamente con la información que se encuentra registrada en el sistema con la familia a la que pertenece la última siembra o cosecha registrada, el usuario debe escoger un mes para que con base en ello el sistema tome la decisión de cuál será el cultivo, la siguiente información será referente a la decisión del sistema de cuál es el cultivo siguiente, una vez que el sistema analizó las familias de los últimos cultivos, determina a que familia debe pertenecer el siguiente cultivo y se muestra en este campo, finalmente una vez determinada la familia a la que pertenece el siguiente cultivo y el sistema determina el o los cultivos

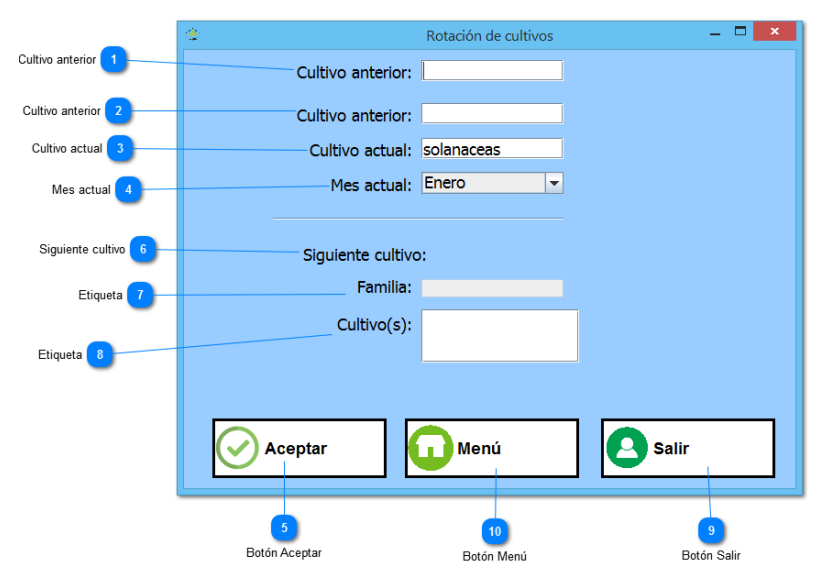

Figura 14 Rotación de cultivos

Fuente: Elaboración Propia

Módulo de historial, este módulo contiene el historial del cultivo, muestra todas las siembras y cosechas registradas, los campos id, cultivo, fecha siembra, fecha cosecha se rellenan de manera automático, algún campo, se muestra la información en la parte inferior de la pantalla, en caso de que la cosecha aún no se registre envía un mensaje "Sin registro". Ver figura 15.

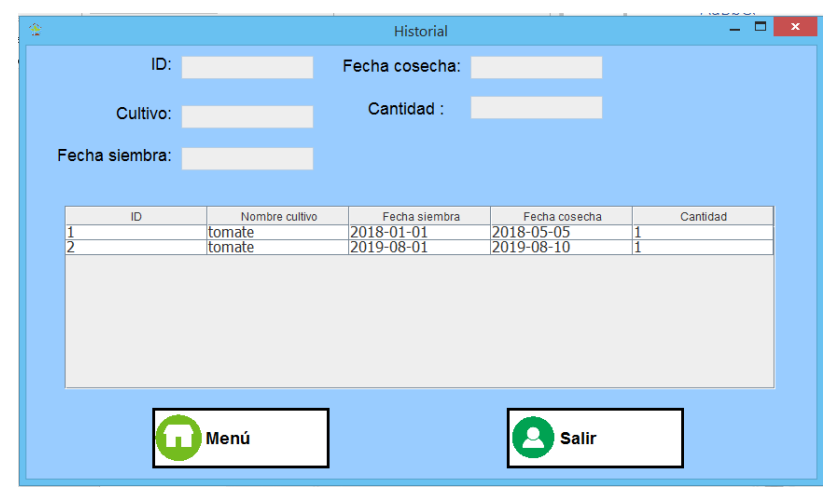

Figura 15 Historial del cultivo Fuente: Elaboración Propia
Módulo de riego, este módulo permite mostrar los riegos ya almacenados en la base de datos. El Tiempo de Riego Diario (T.R.D.) está calculado por la siguiente ecuación:

T.R.D $=\frac{\text { D.B.C. }}{\text { n.g.x qg }}$

Donde:

D.B.C. $=$ Demanda Bruta del Cultivo

n.g. = Número de goteros (2)

$\mathrm{qg}=$ Caudal del gotero (2.3IIh) (Comisión Nacional de Riego, 2000), ver figura 16.

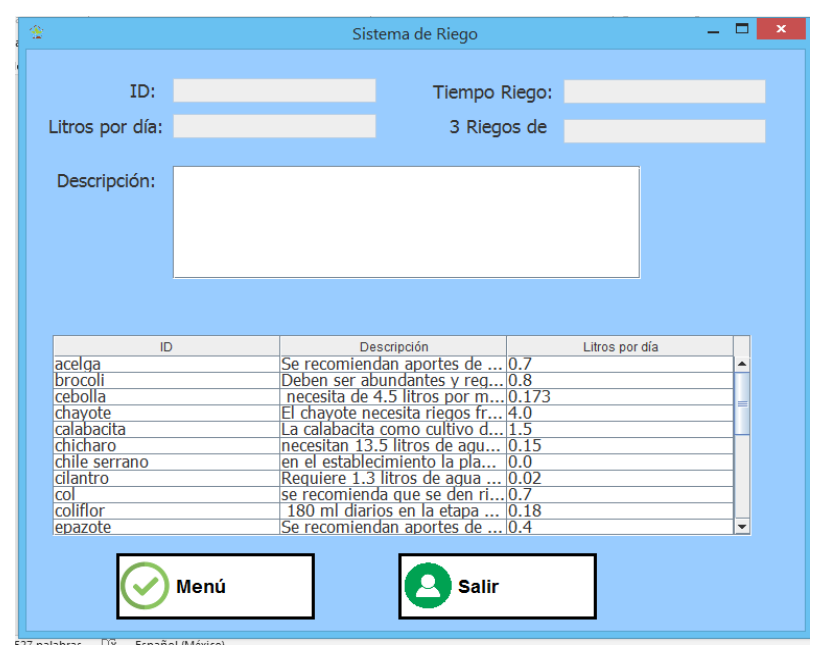

Figura 16 Módulo de riego

Fuente: Elaboración Propia

De esta manera se dividen en tres, para hacer un riego por la mañana, uno por la tarde y otro por la noche._La figura 17 muestra el módulo de bitácoras, en donde el usuario puede insertar una nueva bitácora, el campo cultivo se rellena automáticamente y el campo proceso se elige un tipo de procesos, los cuales son: fertilización, riego, Preparación de abono, siembra, cosecha, corte.

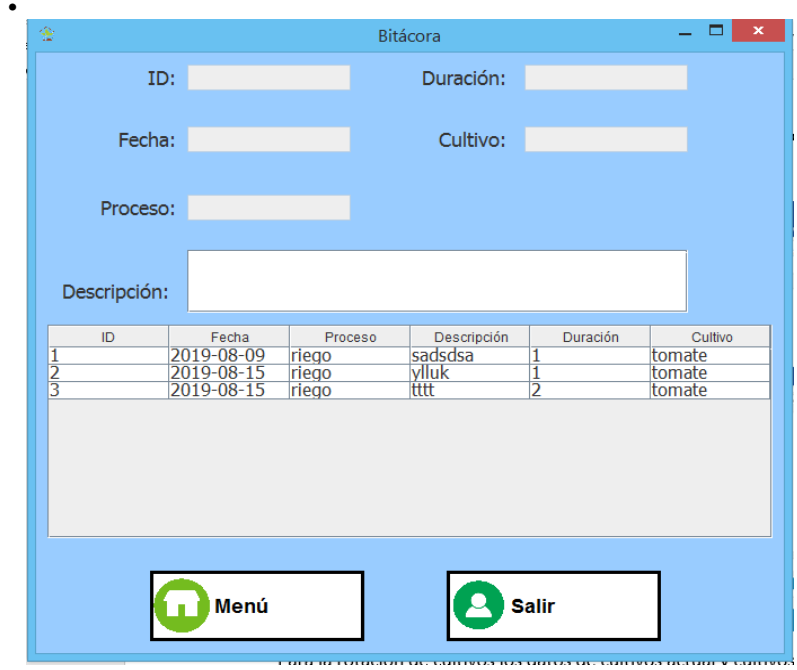

Figura 17 Módulo de bitácora

Fuente: Elaboración Propia

RAFAEL-PEREZ, Eva, MORALES-HERNANDEZ, Maricela, REYESMORALES, Idalia Sarai y NAVARRETE-INFANTE, Néstor Manuel. Sistema Experto para la Rotación de Cultivos en un Invernadero. Revista de Ingeniería Innovativa. 2019. 
Módulo de humedad y temperatura. Como se muestra en la figura 18, contiene los campos de humedad donde el termómetro cambiará dependiendo del porcentaje de humedad que se está detectando, así como el porcentaje de humedad actual, en el campo temperatura, este cambiará dependiendo de los grados centígrados de temperatura que se está detectando y los grados de temperatura actual que tiene el invernadero.

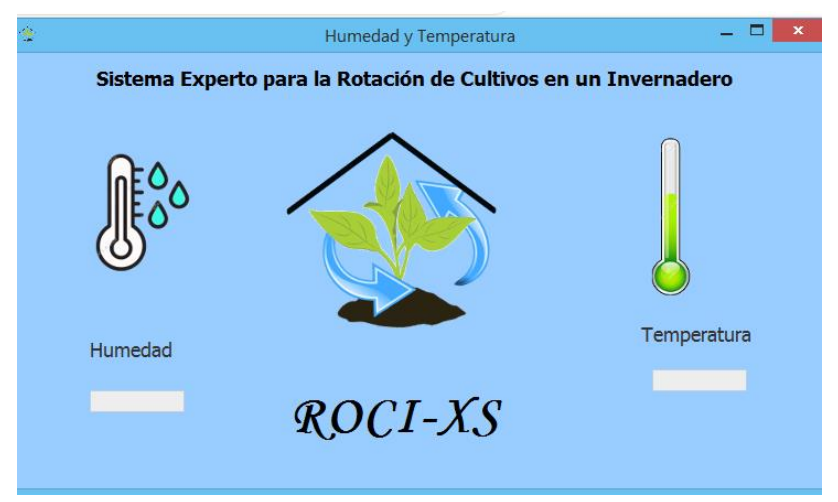

Figura 18 Variables de temperatura y humedad Fuente: Elaboración Propia

\section{Conclusiones}

El proyecto de rotación de cultivos para un invernadero apoyado en un sistema experto fue desarrollado con el objetivo de tomar la decisión del tipo de cultivo idóneo en el invernadero "San Sebastián", que trae los siguientes beneficios: Con la toma de decisión del tipo de cultivo a sembrar en el invernadero el riesgo de pérdida de producción se reduciría considerablemente.

Al desarrollar un sistema experto capaz de tomar la decisión de la rotación de cultivo, ayuda al productor a saber cuál es el cultivo idóneo a sembrar según la experiencia de un experto en la rama; en pro del propio cultivo como del productor, pues al productor se le asegura de esta manera que su cosecha obtendrá la mejor producción según la experiencia y conocimiento del experto $\mathrm{y}$, por otro lado, el cultivo también será beneficiado ya que reduce la incidencia de plagas y enfermedades, al interrumpir sus ciclos de vida; ayuda a disminuir los riesgos económicos, en caso de que llegue a presentarse alguna eventualidad que afecte alguno de los cultivos, finalmente permite balancear la producción de residuos, ya que se pueden alternar cultivos que producen escasos residuos con otros que generan gran cantidad de ellos (CIMMYT-SAGARPA, 2019)

\section{Referencias}

Bastida - tapia A, J.A Ramírez - Arias 2008 Los invernaderos en México Universidad Autónoma de Chapingo Chapingo, México. 123 pág.

Castellanos, J. 2007. Perspectivas de la agricultura protegida en México. In: Segunda Reunión Nacional de Innovación Agrícola y Forestal. Guadalajara, México. Disponible en: http://www.rniaf.org.mx/2007/memoria/ ponencias/protegida/p4_perspectivas1.pdf

Collado, J. A. (s.f.). La Universidad Virtual. Recuperado el 24 de Septiembre de 2014, de Concepto de Sistema de Información en la organización:

http://dpacomputacionunpa.files.wordpress.com /2011/10/1_conceptos_de_si_en_organ izaciones.pdf.

Gabino Alberto Martínez-Gutiérrez*, René Díaz-Pichardo, Griselda Juárez-Luis, Yolanda D. Ortiz-Hernández, Juana Y. López-Cruz. Caracterización de las unidades de producción de tomate en invernaderos de Oaxaca Instituto Politécnico Nacional. CIIDIR Unidad Oaxaca, Hornos 1003, C.P. 71230, Santa Cruz Xoxocotlán. Oaxaca. México

Gary W. Hansen, J. V. (2014). Diseño y Administracion de Bases de Datos. Mexico: Prentice Hall.

Juárez López Porfirio, Bugarín Montoya Rubén, Castro Brindis Rogelio, Sánchez Monteón Ana Luisa, Cruz Crespo Elia, Juárez Rosete Cecilia Rocío, Alejo Santiago Gelacio, Balois Morales Rosendo. Estructuras utilizadas en la agricultura protegida.

Unidad Académica de Agricultura, Universidad Autónoma de Nayarit, Departamento de Fitotecnia, Universidad Autónoma Chapingo

Laudon, K., \& Laudon, J. (2004). Sistemas de información gerencial (Octava ed.). México: Pearson

Moreno Reséndez, Alejandro; Aguilar Durón, Juanita; Luévano González, Armando Características de la agricultura protegida y su entorno en México Revista Mexicana de Agronegocios, núm. 29, julio-diciembre, 2011, pp. 763-774 
Sociedad Mexicana de Administración Agropecuaria A.C. Torreón, México

Nevado, M. (2010). Introducción a Las Bases de Datos Relacionales. España: Visión Libros 